\title{
APPLICATION OF GENE EXPRESSION DATA AND CELLULAR AUTOMATA TO PREDICT DISEASE PROGRESS IN A CARDIAC HYPERTROPHY MODEL
}

\author{
A.K. MACPHERSON ${ }^{1}$, L.M. CROSBY ${ }^{2}$ \& P.A. MACPHERSON ${ }^{3}$ \\ ${ }^{1}$ Institute for Biomedical Engineering \& Mathematical Biology, Lehigh University Bethlehem, PA, USA. \\ ${ }^{2}$ GenExpressions, Inc, Raleigh, NC, USA. \\ ${ }^{3}$ Department of Applied Technology, Rogers State University, Claremore, OK, USA.
}

\begin{abstract}
The discernment of gene expression changes at the molecular level presents great opportunities to conquer human disease; therefore it is important that these data find use in clinical practice. Here an example is given of how gene expression data may be used in the prediction of the severity of heart disease leading to cardiac hypertrophy (enlargement of heart muscle). Using a mathematical modeling approach based on cellular automata and available temporal expression gene profiles, the rate of development of hypertrophy in cardiomyocytes was studied. In the model, during 5\% of the time transcriptional activation of genes resulted in significant increases in heart muscle mass. For hypertrophy originating in one or a few cells, the spread of the lesion occurs as a result of the intercellular transmission of information from the 'seed' cells to neighbors. Model output indicated that signal transmission time correlated with net increases in cardiomyocyte mass. There was a threshold signal time of 12 minutes, below which net mass increase was negligible. This implies that certain persons with a tendency to short transmission times would probably not suffer heart damage even while experiencing limited heart muscle growth, in the event of hemodynamic overload. We hypothesize that anti-hypertrophic drugs actually reduce the transcellular signal transmission time, resulting in reduced incidence/severity of heart disease.

Keywords: angiotensin II, $\alpha$-actin, anti-hypertrophic drug action, cellular automata, gene expression, left ventricular hypertrophy, left ventricular mass increase, MHC- $\beta$, myosin light chain, TGF $\beta$.
\end{abstract}

\section{INTRODUCTION}

Many diseases such as cancer, cardiac hypertrophy, Alzheimer, diabetes and so on arise from genetic changes. As only certain people contract a given disease there must be factors that initiate the onset of the disease. Further, only in certain cases does the disease spread. The initiation and spread will be disease dependent and the present paper shows how typically the process may work. The example discussed here is how gene expression data may be used in the prediction of the severity of heart disease leading to cardiac hypertrophy. The advent of large-scale differential gene expression array technology (LSDGE) is making massive data sets available for detailed examination. These data can be useful in clinical practice to predict disease development. We chose to model the development of cardiac hypertrophy, the enlargement of heart muscle, using available gene expression data. Cardiac hypertrophy, although an adaptive change that can be beneficial when limited, has many serious consequences when uncontrolled, resulting in arrhythmias and ischemic heart disease. Specific changes in cardiomyocytes during the development of cardiac hypertrophy include the induction of 'immediate-early' genes (heat shock protein genes and proto-oncogenes), changes in gene expression (re-expression of fetal genes) and increased protein synthesis [1]. The first step in the progress towards lesion development is believed to be the transcription of a gene responding to a provocative stimulus, producing a messenger RNA (mRNA) followed by translation into a protein or proteins. Proteins frequently act as signal transduction molecules, transmitting signals within and between cells. In response to mechanical stress resulting in the stretching of myocytes, signals may be generated that direct cardiomyocytes to increase their size without dividing, i.e. hypertrophy. While originating in a few cells, this signal may eventually be transmitted through cascade amplification to 
all cells in the tissue, finally affecting the entire organ. For a complete review of the mechanosensors and signal transduction pathways believed to participate in the development of cardiac hypertrophy, see [1].

Enlargement of the left ventricle of the heart with a resultant increase in heart muscle mass, which can be due to hemodynamic overload, is known as left ventricular hypertrophy (LVH). The expression or release of several growth factors and hormones within myocytes comprising the heart muscle wall is known to be involved indirectly in cardiac hypertrophy, among them angiotensin II (Ang II), endothelin I and transforming growth factor $\beta$ (TGF- $\beta$ ) [2]. Since 1934, increases in blood pressure have been known to occur as a result of the release of the hormone Ang II [3] in humans, and Ang II produces myocyte enlargement within the left ventricle [4]. Ang II stimulates myocytes by binding to its cognate (G-protein coupled) receptors on myocyte plasmalemma surfaces and initiating signal cascades within the cells. Myocytes have intimate cell-cell contact with one another and possess gap junctions consisting of intercalated discs (desmosomes) capable of passing signals rapidly from cell to cell. Electrical coupling through gap junctions synchronizes the contractions of heart muscle cells. The myocytes are in sheets approximately parallel to the inside surface of the ventricle wall for a considerable distance into the myocardium [5] forming a functional syncytium [6]. The electrical signals propagate rapidly in the parallel direction by cell-cell communication through the gap junctions, while in the direction normal to the sheets of cells, electrical signals are transmitted by ionic conduction. In lieu of specific knowledge concerning the transmission of mRNA-generated signals, the latter are here assumed to follow paths similar to the electrical signals. Myocyte contractile proteins, cardiac muscle thin actin and thick myosin filaments are arranged in orderly arrays of myofibrils forming contractile sarcomeres and generating a striated appearance. The sarcomeres are covered by the membranous sarcolemma and surrounded by a membranous network, the sacroplasmic reticulum that abuts a transverse T-tubule network. The latter is composed of structural proteins formed from invaginations of the plasma membrane. Action potentials resulting in muscle contraction are transmitted via the T-tubule network to the sarcoplasmic reticulum, where opening of $\mathrm{Ca}^{2+}$ release channels is triggered [2].

Ang II influences the cell by interacting with receptors on the myocyte surface, leading to an increase in the number of contractile protein units, and thus an increase in sarcomere length. This results in an increase in cell size, which occurs without division, and brings about an increase in left ventricular mass (LVM), one of the few measurable quantities involved with the early stages of the disease. Drugs known as angiotensin receptor blockers prevent the development of the disease by allosteric inhibition, docking at different sites on the receptor than does Ang II.

Left ventricular hypertrophy has been linked to upregulation of expression of certain fetal genes $[7,8]$. The study of this process, termed 'gene reprogramming', has been mainly based on experiments with small animals. The period of development of cardiac hypertrophy in laboratory animals may or may not be equivalent to that in humans, which occurs over many years [9]. In humans, the available data have been generally limited to end-stage heart failure (as stated above), and thus there is some uncertainty as to which genes are the most significant in the early development of hypertrophy in humans.

In order to model the development of LVH, one must know the particular genes involved and the amount of involvement of each of those genes. A survey of several genes whose upregulation is known to be associated with cardiac hypertrophy provides some values. $\beta$-myosin heavy chain ( $\beta$-MHC) was shown to be upregulated [10] in $30 \%$ of cases, myosin binding protein-C in $20 \%$, cardiac troponin $\mathrm{T}$ in $20 \%$ and cardiac $\alpha$-actin in $<5 \%$.

There are many unknowns during LVH. The chemistry and molecular mechanism involved in the increase in myocyte mass has not been determined. At the inner surface of the heart wall, the 
endocardium, the myocytes are oriented parallel to the wall, with little room for expansion in a lengthwise direction. The interstitial fluid occupies approximately $30 \%$ of the interstitial volume at the endocardium [5] and the volume decreases across the myocardium. Following the assertions presented in [11] the myocytes will likely expand mainly in the lateral direction. Expansion along the myocyte length would increase the stresses in the wall and cause hypertrophy more quickly. The increase in mass of the four proteins mentioned above is assumed to occur in a particular order. To allow the inner components to increase in size, the sarcoplasmic reticulum must expand first. Again, following the assertions concerning embryonic development [11], the genes responsible for membrane development are assumed to be upregulated initially, while the genes involved with other processes will remain dormant. Other genes will be upregulated as required to increase the mass of the sarcolemma, T-tubules and finally the whole myocyte. A sequential upregulation in the involved components is postulated. The values found $[7-9,12]$ represent those measured at the end stages of disease progression and probably only indicate that these genes have been involved in gene reprogramming at some earlier time. For example, the upregulation of cardiac $\alpha$-actin is found to occur in only $5 \%$ of occasions at late stages. If it were responsible for the increase in the outer membrane, it would have been more highly upregulated in the early stages, but downregulated to normal levels before the final stage.

The cells stimulated by increased Ang II expression transmit information to other cells either through the intercalated discs that join the ends of the cells or through the lateral membranes and across the interstitial fluid that surrounds the cells. There are branches between the sheets of myocytes, but these will be ignored in the present simplified model. The development of the first model of cellular automata is credited to John von Neumann in the late 1940s [13]. A recent description of the method as presented in [11] will be used. As found therein, the results are obtained using a few simple rules and are independent of many of the otherwise seemingly complex assumptions.

\section{METHOD}

The first step was the modeling of selected gene expression changes within the cell. The time over which gene expression data are available is usually limited. In order to estimate the changes in mRNA transcript levels over times longer than those measured to date, a system of equations was created. There are many possible techniques for modeling gene expression time series data. It was decided to treat this series as a linear dynamical system (LDS). The observed values of gene expression levels and other known variables, at various times, are used to calculate the constants that apply to the estimation process. There may be other unknown genes that influence the time development of the system, as addressed below. An LDS system [14] can be described by the following equations:

$$
\begin{gathered}
x_{t+1}=A x_{t}+B u_{t}+w_{t} \\
G_{t}=C x_{t}+v_{t}
\end{gathered}
$$

The values of $x_{t}$ are the mRNA transcript levels at time $t$. The values of $u_{t}$ are those of unknown mRNA transcripts, which are not those of the specified genes but are believed to influence the result. The values of $x_{t}$ are used to calculate the matrices $A, B, C, D$ and $u_{t}$ using Kalman filtering [15]. The values of the transcript levels at later times $\left(g_{t}\right)$ can be calculated using eqns (1) and (2). As there is a certain level of noise in all measurements, state variable noise $w_{t}$ and output noises $v_{t}$ were included in the model. These are zero-mean random vectors. The matrix $A$ is the transition state matrix $B$ is the input to the state matrix, and $C$ is the state-to-observation matrix. The matrices $A, B$ and $C$ are derived using Kalman filtering [15] and the gene expression data. Once the matrices are determined, 
estimates concerning the future development of the system can be made. The method has been previously described $[14,16]$.

The next events to occur are those molecular processes known to be involved with the initiation of relevant protein translation within the myocyte. Most of the research on the molecular mechanisms of hypertrophy has been undertaken on neonatal rats and is poorly understood [9]. As an example of the potential drawbacks associated with the use of neonatal rats, Ang II activates the gene $\mathrm{p} 70^{\mathrm{S} 6}$ kinase; however $\mathrm{p} 70^{\mathrm{S} 6}$ kinase was not found to be involved in gene reprogramming. Ang II stimulated c-fos and c-jun in this system but not in adult rat hearts [9]. Therefore, such data must be interpreted with caution.

Last of all, a model network for the transfer of information from cell to cell is required. The initial signal is received via the receptors on the surface of the cells. The fraction of receptors that are occupied during the early portion of diastole can be calculated using a combination of Monte Carlo and molecular dynamics methods [17]. Thus, for a specified level of Ang II mRNA, the expected number of receptors occupied can be calculated. Using the methods of cellular automata, the development of hypertrophy can then be followed. This requires the development of rules based on the methods in [11]. The cells and interstitial spaces are represented in the computer by cell boxes aligned on a twodimensional grid. The state of each cell is altered by the conditions of its surrounding cells at the previous iteration, to simulate the transmission of a signal through the cell tissue. The five states and transitions are as shown in Fig. 1.

\section{RESULTS}

The two-dimensional model used here has one axis (referred to as the horizontal axis) along the endocardium and the vertical axis penetrates into the myocardium, normal to the endocardium. In order to establish the probability functions for the application of the network solutions, it is necessary to derive the probability of a myocyte being stimulated by Ang II. It is known that the level of Ang II in the blood of healthy individuals is about $5 \mathrm{fmol} / \mathrm{mg}[18,19]$. The number of receptors measured is approximately $7.75 \mathrm{fmol} / \mathrm{mg}$ [20], and the volume of a myocyte $20 \times 10^{3} \mu \mathrm{m}^{3}$ [21]. Thus, the number of receptors on one side of a myocyte is $5 \times 10^{6}$, assuming a roughly cuboidal shape. It is known that at the normal level of Ang II in a healthy human heart, hypertrophy usually does not occur. Using the techniques described in [11], the fraction of receptors occupied on each myocyte throughout diastole was calculated. It is assumed that Ang II molecules bound to the receptors during diastole mechanically dissociate during systole. This is because the myocardium is squeezed tightly during the later part of the diastolic and systolic phases. Therefore, mechanical pressure is believed to contribute to the physical dissociation of Ang II ligands from their receptors. Only about 0.4 seconds of a normal heartbeat is, therefore, available for binding of the Ang II ligands to their receptors.
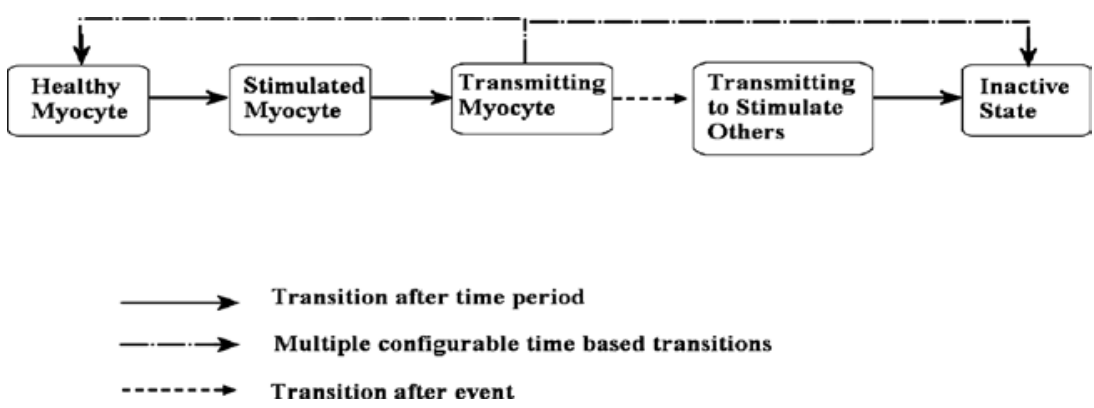

Figure 1: Transition states for stimulated myocytes. 
If the chemistry and biological mechanisms involved with different gene upregulations were known, the effect of the various gene expression changes on the appropriate tissues could be determined. At present these mechanisms are unknown, therefore for this particular problem, four genes that are known to be involved have been selected. Myosin is required during the cellular mechanics of contraction and growth, and a possible relation between the three genes (for derivation see below) would be as shown in Fig. 2a. The unknown inputs were chosen for the ith time step as:

$$
u(i)=2+\text { random number }
$$

Thus the strength of the input increases with time. Due to the nature of the probability functions involved with the calculation, the results are different each time the program is run.

Running the program 100 times yields a typical result as shown in Fig. 2a. This represents a typical disease end-stage event, as myosin is upregulated and contributes structurally and mechanically to cell enlargement, as previously mentioned. Upregulation occurs within the first 100 time steps and different genes are upregulated in different runs. After a short time period, all transcript levels assume a constant value. Usually when there is little change in expression of a gene, the signal is very noisy as shown in Fig. 2a. The effect on gene reprogramming is determined, depending on how long the unknown inputs are allowed to operate. Thus the 'unknown inputs' are regulatory factors within the cell that can stimulate transmission.

However, on about $5 \%$ of occasions the change in the genes is as shown in Fig. 2b. Here gene expression varies in a specific direction over time, approaching a very high or very low value, rather than displaying a random variation that has approximately the same average value during the course of the run. These cases would therefore be expected to display sufficient changes in gene expression magnitude to initiate LVH. In addition the changes have a smaller variation due to noise, and are

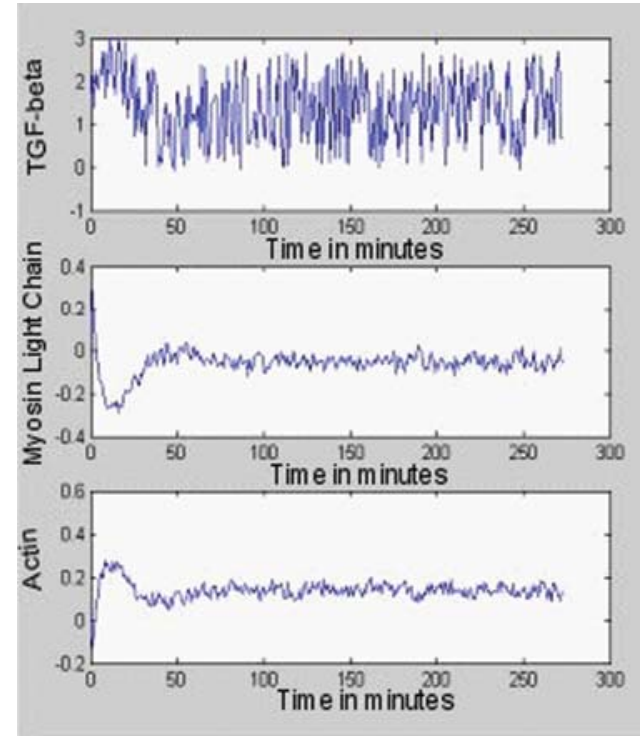

(a)

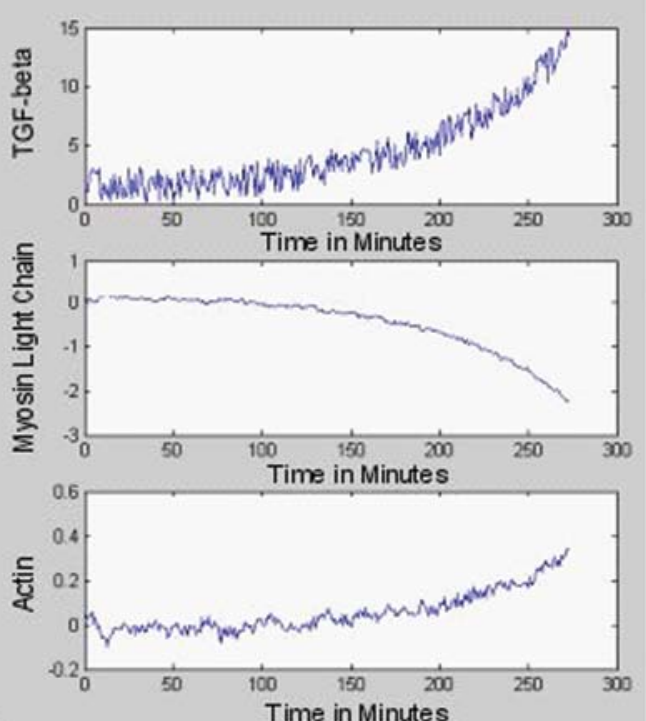

(b)

Figure 2: (a) Graph showing typical time variation of gene expression signal intensities when the unknown inputs are as shown in eqn (1). (b) Time variation of gene expression signal intensity during $5 \%$ of all iterations run. 
larger in magnitude versus time than the typical results shown in Fig. 2a. The expression changes indicated in Fig. $2 \mathrm{~b}$ may represent the driving forces of hypertrophic change in cardiac myocytes. Thus, in about $95 \%$ of instances, successful receptor binding of Ang II may not alter gene expression or protein structure within neighboring, signaled cardiomyocytes.

Calculations using the rules of cellular automata are performed to model the progress of the disease, after the initiation of gene reprogramming. Initially each cell, $P$, with $i=$ row, $j=$ column, is healthy. It will transition to the 'stimulated' state either by a random stimulus as follows:

$$
P_{(i, j)}=P_{\text {random_infection_rate }} \frac{i^{4}}{\text { total_rows }^{4}}
$$

or by a transmission from its neighbors as shown in eqn (5).

$$
P_{(i, j)}=\frac{i^{2}\left(\text { HorizontalTransRate }\left(T_{(i-1, j)}+T_{(i+1, j)}\right)+\operatorname{VerticalTransRate}\left(T_{(i, j-1)}+T_{(i, j+1)}\right)\right)}{\text { total_rows }^{2}}
$$

where $T_{(i, j)}$ indicates whether the cell is transmitting (state 1) or not (state 0 ) at the previous turn. After a user-specified time period, a cell moves automatically from stimulated to transmitting, i.e. it may begin to send its signal to its neighbors. In this model, it will transmit for a specified time period after which it will transition either back to quiescent, or if it successfully stimulates a neighboring cell, it will become 'terminal'. The latter is representative of a state where no further transitions are possible.

Associated with each cell is a counter, and state variables for the previous and next states. During each iteration, every cell is checked to determine its transitions, based upon its counter for timebased transitions or its neighbors' previous states. There is a bias factor included in the calculation of the transmission and random stimulation. The latter two are biased in favor of transitions (signals) towards the myocytes near the endocardium. To evaluate the probabilistic transitions, a pseudorandom number is generated from a uniform distribution and compared to the appropriate probability threshold. If the random value is less than the probability, then the event is considered to have occurred and the next state variable is set with the counter. Applying these rules and evaluating the expressions over time, eventually a stable state is reached. Hence the increase in left ventricular mass was found to be mainly a function of the time a cell was allowed to transmit. The values used were:

- Initial seed $=0.35$

- Probability of transmission into the myocardium $=0.1$

- Probability of transmission parallel to the endocardium $=0.01$

- Probability of random stimulation $=1.0 \mathrm{e}-06$

- Period before transmission $=5$ time steps

- Period between endocardium stimulation $=10$ time steps.

From these initial values, it is calculated that in order to create a significant change in LVM within a year, the time steps would need to be at least approximately 12 minutes long. If the period between successful transmissions at the layer of cells closest to the endocardium was reduced, then this layer became effectively 'sealed' and the infection was not transmitted further into subsequent layers of myocardiocytes. Fig. 3 shows percentage increase in LVM after the transmission scenario has been operative for one year, for time steps from zero to 80 .

It can be seen that when the transmitting times are less than 12 minutes, a negligible increase in LVM occurs. However, if the cells are allowed to transmit for a greater length of time, the increase in LVM will be significant. 


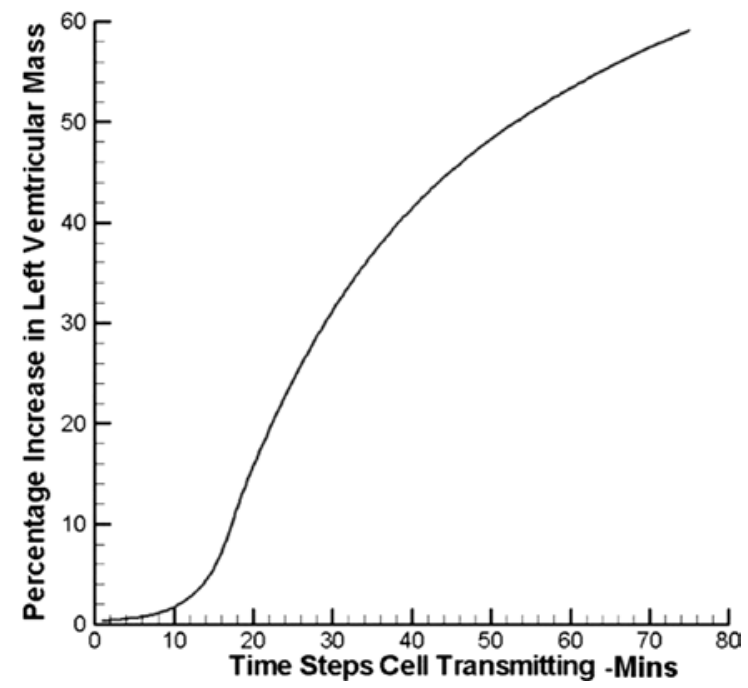

Figure 3: A snapshot showing the predicted percentage increase in left ventricular mass ( $y$-axis) after one year using various time steps ( $x$-axis). It can be seen that for time steps of 12 minutes or greater, the increase in LVM rises steeply, possibly contributing to the development of LVH.

\section{DISCUSSION}

The current study indicates that LVH may have a gene expression-associated stochastic basis, linked to the transmission time of cell-cell signaling. Since it is known that a reduction in the levels of Ang II can be achieved by blocking the transformation of the Ang II precursor into Ang II by using angiotensin converting enzyme inhibitors (ACEs), it may be supposed that if ACE inhibitors are not used, an increase in Ang II may occur in patients that eventually develop LVH. The greater the ambient amount of Ang II within the cells, the greater will be the likelihood of gene upregulation, as in Fig. 2b. The latter, being associated with signal transduction, might permit increased cell-cell signal transmission time, increasing the effective time step of the model. As regards the determination of a 12-minute or greater signal threshold, it may be relevant that [22] it has been shown that cells grow for 12 minutes followed by 12 minutes of rest. In the case of cells signaling for greater than 12 minutes, a disruption of the cell's normal growth cycle could ensue, and this last could have a dysregulatory effect on other cellular homeostatic mechanisms, including those relating to gene expression control.

Based on model output, the probability that an increase in Ang II will result in significant gene upregulation is relatively small. The upregulation is dependent on the inputs, which are different from person to person due to phenotypic variation. This may explain the fact that Ang II could increase for a period of time, while not resulting in physical damage to the heart. If the system did not respond this way, the incidence of heart damage would be larger than is actually found in the general population. In addition, it presents a plausible explanation for why some patients with hemodynamic overload do not suffer heart damage, i.e. even if the genes are upregulated significantly, heart damage will not occur if the transmission time between cells is below a critical value. It is hypothesized that the ACE drugs work not only by receptor blocking, but also by reducing the transmission time below the critical 12-minute limit. 
The usefulness of constructing a hypothesis to fit known facts without the required experimental results has many examples in science, a notable one being on the variation in the permeability of axon membranes [23]. Fully eight years before their discovery, Hodgkin and Huxley proposed the existence of voltage-gated ion channels, based solely upon modeling results obtained using available observations.

Not only must gene upregulation be studied, however in addition, the effect of the foregoing on cell structural changes and growth, and eventual cell-cell signaling is of vital importance. We believe the transmission time of the gene expression signal from cell to cell will prove to be crucial in the use of this information to change treatment outcomes clinically [24]. To this end, in the future, physiologic function testing or genetic profiling may be combined with gene expression data in order to make use of LSDGE technology in the clinical setting.

\section{ACKNOWLEDGEMENTS}

The authors wish to acknowledge the help of the many individuals who contributed, directly or indirectly, to this work. This work was supported by Lehigh University.

\section{REFERENCES}

[1] Ruwhof, C. \& van der Laarse, A., Mechanical stress-induced cardiac hypertrophy mechanisms and signal transduction pathways. Cardiovascular Research, 47, pp. 23-37, 2000. doi:10.1016/ S0008-6363(00)00076-6

[2] Alberts, B., Johnson, A., Lewis, J., Raff M. \& Walter, P., Molecular Biology of the Cell, 4th edn, Garland Science/Taylor \& Francis Group: London, 2000.

[3] Goldblatt, H., Lynch, J., Hanzel, R.F. \& Summerville, W.W., Studies on experimental hypertension: I The production of persistent elevation of systolic blood pressure by means of renal ischemia. J. Exp. Med., 59, pp. 347-349, 1934. doi:10.1084/jem.59.3.347

[4] Dzau, V.J. \& Gibbons, G.H., Autocrine and paracrine mechanisms of vascular myocytes in systemic hypertension. Am. J. Cardiol., 60, pp. 991-1031, 1987. doi:10.1016/00029149(87)90468-1

[5] LeGrice, I.J., Slaill, B.H., Chai, L.Z., Edgar, S.G., Gavin, J.B. \& Hunter, P.J., Laminar structure of the heart: ventricular myocyte arrangement and connective tissue architecture in the dog. Am. J. Physiol., 269(2 Pt 2), pp. H571-H582, 1995.

[6] Marshall, J.M., The heart. Medical Physiology, 13th edn, ed. V.B. Mountcastle, Mosby: New York, 1974.

[7] Parker, T.G., Molecular biology of myocardial hypertrophy and failure: gene expression and trophic signaling. New Horizons, 3, pp. 288-300, 1995.

[8] Schwartz, K., Chassagnw, C. \& Boheler, K.R., The molecular biology of heart failure, JACC, 22(4 Suppl A), pp. 30A-33A, 1993. doi:10.1016/0735-1097(93)90459-E

[9] Kim, S. \& Iwao, H., Molecular and cellular mechanisms of angiotensin II-mediated cardiovascular and renal diseases. Pharmacological Reviews, 52(2), pp. 11-34, 2000.

[10] Marian, A.J. \& Roberts, R., The molecular genetic basis for hypertrophic cardiomyopathy. J. Mol. Cell. Cardiol., 33, pp. 655-670, 2001. doi:10.1006/jmcc.2001.1340

[11] Wolfram, S., A New Kind of Science, Wolfram Media, Inc.: Champagne, Illinois, 2002.

[12] Lim, D.-S., Roberts, R. \& Marian, A.J., Expression profiling of cardiac genes in human hypertrophic cardiomyopathy: insight into the pathogenesis of phenotypes. JACC, 38(4), pp. 1175-1180, 2001. doi:10.1016/S0735-1097(01)01509-1

[13] Poundstone, W., The Recursive Universe: Cosmic Complexity and the Limits of Scientific Knowledge, Oxford University Press: Oxford, 1987. 
[14] Rangel, C., Wild, D.L. \& Falciani, F., Modelling biological responses using gene expression profiling and linear dynamical systems. Proceedings of the 2nd International Conference on Systems Biology, OmniPress: Madison, WI, pp. 248-256, 2002.

[15] Grewal, M.S. \& Andrews, A.P., Kalman Filtering, Theory and Practice Using MATLAB, John Wiley: New York, 2001.

[16] Ghahramani, Z. \& Hintin, G.E., Parameter Estimation for Linear Dynamical Systems, University of Toronto Technical Report, CRG-TR-96-2, 1996, http://www.gatsby.ucl.ac.uk/ zoubin [2003].

[17] Macpherson, A.K. \& Neti, S., The effect of angiotensin II on heart blood flow and hypertension. Advances Fluid Mechanics IV, eds M. Rahman, R. Verhoeven, C.A. Brebbia, WIT Press: Southampton, pp. 1-12, 2002.

[18] Schuijt, M.P., de Vries, R., Saxena, P.R., Schalekamp, M.A. \& Danser, A.H., Vasoconstriction is determined by interstitial rather than circulating angiotensin II. Br. J. Pharmacol., 135(1), pp. 275-283, 2002. doi:10.1038/sj.bjp.0704452

[19] Schuijt, M.P., van Kats, J.P., de Zeeuw, S., Dunker, D.J., Verdouw, P.D., Schalekamp, M.A.D.H. \& Jan Danser, A.H., Cardiac interstitial fluid levels of angiotensin I and II in the Pig. Journal of Hypertension, 17(12 Part 2), pp. 1885-1891, 1999. doi:10.1097/00004872-199917121-00017

[20] Zisman, L.S., Asano, K., Dutcher, D.L., Ferdensi, A., Robertson, A.D., Jenkin, M., Bush, E.W., Bohlmeyer, T., Perryman, M.B. \& Britow, M.R., Differential regulation of cardiac angiotensin converting enzyme binding sites and $\mathrm{AT}_{1}$ receptor density in the failing human heart. Circulation, 98(17), pp. 1735-1741, 1998.

[21] Kajstura, J., Zhang, X., Liu, Y., Szoke, E., Cheng, W., Olivettis, G., Hintze, T.H. \& Anversa, P., The cellular basis of pacing-induced dilated cardiomyopathy. Circulation, 92, pp. 2306-2317, 1995.

[22] Morré, D.J., Chueh, P.-J., Pletcher, J., Tang, X., Wu, L.-Y. \& Morré, D.M., Biochemical basis for the biological clock. Biochemistry, 41(40), pp. 11941-11945, 2002. doi:10.1021/bi020392h

[23] Hodgkin, A.L. \& Huxley, A.F., A quantitative description of membrane current and its application to conductance and excitation in nerve. J. Physiol., 117, pp. 500-554, 1952.

[24] Streeter, D.D., Powers, W.E., Ross, M.A. \& Torrent-Guasp, F., Three-dimensional fiber orientation in the mammalian left ventricular wall. Cardiovascular System Dynamics, eds J. Baan, A. Noordergraaf \& J. Raines, MIT Press: Cambridge, MA, pp. 73-84, 1978. 